\title{
Ratio and time requirements on operant schedules: effort- related effects of nucleus accumbens dopamine depletions
}

\author{
Susana Mingote, Suzanne M. Weber, Keita Ishiwari, Mercè Correa* and John D. Salamone \\ Department of Psychology, University of Connecticut, Storrs, CT 06269-1020, USA
}

Keywords: activation, decision making, instrumental conditioning, motivation, rat, reinforcement, reward

\begin{abstract}
Accumbens dopamine (DA) depletions produce deficits that are related to the ratio requirement of the operant schedule; however, it is also possible that time without reinforcement is a factor. The present study examined the effects of accumbens DA depletions in rats using variable interval $(\mathrm{VI})$ schedules with additional fixed ratio (FR) requirements. Four VI schedules were used (VI 60/FR 1, VI 120/FR 1, VI 60/FR 10, VI 120/FR 10). Attachment of the additional work requirement increased response rates under control conditions. After surgery, there was no interaction between interval level (i.e. 60 vs. $120 \mathrm{~s}$ ) and DA depletion, but there was a significant interaction between ratio requirement (i.e. 1 vs. 10) and DA depletion within the first week after surgery. DA depletions substantially impaired performance on the schedules with added FR 10 requirements, an effect that was largely dependent upon a reduction in fast responses (i.e. interresponse times less than $1.0 \mathrm{~s}$ ). There was little effect of DA depletion on overall responding on VI 60/FR 1 and VI 120/FR 1 schedules. DA depletions also increased the tendency to take long pauses in responding (i.e. > 20.0 s), and this effect was evident across all schedules tested. Thus, accumbens DA depletions interact with work requirements and blunt the rate-enhancing effects of moderate size ratios, and also enhance the tendency to pause. Attachment of ratio requirements to interval schedules is a work-related response cost that provides a challenge to the organism, and DA in nucleus accumbens appears to be necessary for adapting to this challenge.
\end{abstract}

\section{Introduction}

The regulation of instrumental behavior involves an array of behavioral processes, including primary motivational functions, pavlovian and instrumental associations, and decision making related to economic factors such as cost/benefit analyses and work requirements (Collier \& Jenkins, 1969; Staddon, 1979; Hursh et al., 1988; Salamone, 1991, 1992; Dickinson \& Balleine, 1994; Salamone et al., 1997, 2003; Aberman \& Salamone, 1999; Everitt et al., 1999; Hamill et al., 1999; Bickel et al., 2000; Walton et al., 2002). A complex network of brain circuitry participates in the regulation of instrumental behavior, and the dopamine (DA) innervation of nucleus accumbens is an important contributor to this circuitry (Berridge \& Robinson, 1998; Cardinal et al., 2002; Horvitz, 2002; Salamone \& Correa, 2002; Salamone et al., 2003; Cardinal \& Everitt, 2004; Wise, 2004). Evidence indicates that DA in the nucleus accumbens does not mediate primary food motivation or appetite (Ungerstedt, 1971; Koob et al., 1978; Salamone et al., 1993b; Nowend et al., 2001; Baldo et al., 2002; for reviews see Salamone et al., 1997, 2003; Salamone \& Correa, 2002). Nevertheless, accumbens DA modulates several important aspects of incentive motivation (Salamone, 1991, 1992; Salamone et al., 1997; Berridge \& Robinson, 1998; Ikemoto \& Panksepp, 1999; Kelley, 1999; Berridge, 2000; Cardinal et al., 2002; Di Chiara, 2002; Salamone \& Correa, 2002). Accumbens DA is involved in processes related to responding to conditioned stimuli (Taylor \& Robbins, 1986; Everitt et al., 1989; Cador et al., 1991;

Correspondence: Dr J. D. Salamone, as above.

E-mail: Salamone@psych.psy.uconn.edu

*Present address: Department of Psicologia, Universitat Jaume I, Castello, Spain.

Received 24 July 2004, revised 18 December 2004, accepted 22 December 2004
Parkinson et al., 1999; Cardinal et al., 2002; De Borchgrave et al., 2002), including pavlovian-instrumental transfer (Wyvell \& Berridge, 2000; De Borchgrave et al., 2002; Parkinson et al., 2002). Microdialysis and voltammetry studies reported an increase in accumbens dopamine efflux during presentations of conditioned stimuli, and during lever pressing (Sokolowski et al., 1998; Bassareo \& DiChiara, 1999; Cousins et al., 1999; Datla et al., 2002; Roitman et al., 2004). Recently published electrophysiological studies describe changes in the firing rate of nucleus accumbens neurons that are related to environmental stimuli and the performance of operant responses for food reward (Carelli, 2002; Nicola et al., 2004).

One of the suggested functions of accumbens DA is enabling organisms to overcome work-related response costs in instrumental responding (Salamone et al., 1991, 1997, 2003; Salamone \& Correa, 2002). Interference with accumbens DA alters effort-related decision making in choice procedures that involve allocating responses between various reinforcement values and response costs (Salamone et al., 1991, 1994, 1997, 2003; Cousins et al., 1993, 1996; Cousins \& Salamone, 1994; Sokolowski \& Salamone, 1998; Koch et al., 2000; Nowend et al., 2001). Moreover, the effects of accumbens DA depletions on food-reinforced lever pressing are highly dependent upon the schedule being tested. For example, overall responding on the continuous, or fixed ratio 1 (FR 1), schedule, which is dependent on primary food reinforcement and is sensitive to extinction and reinforcer devaluations such as pre-feeding, is largely unimpaired by accumbens DA depletions (McCullough et al., 1993; Salamone et al., 1995; Aberman \& Salamone, 1999; Ishiwari et al., 2004). Nevertheless, schedules requiring animals to press repeatedly to receive food (i.e. ratios $\geq$ FR 5) are sensitive to the effects of accumbens DA depletions, and the size of the ratio requirement is directly related to 
the degree of impairment produced by DA depletions (Salamone et al., 1993a, 1999, 2001; Aberman et al., 1998; Sokolowski \& Salamone, 1998; Aberman \& Salamone, 1999; Ishiwari et al., 2004).

Several factors, including the work requirement, the rate-enhancing effects of ratios and reliance on conditioned stimuli, may combine to make ratio schedules sensitive to the effects of accumbens DA depletions (Salamone et al., 2001; Correa et al., 2002). Another possible factor is the intermittent nature of reinforcement presentation (Aberman \& Salamone, 1999; Cardinal et al., 2001, 2002; Salamone et al., 2001). Because it takes time to complete the ratio, high ratio schedules impose a time interval between reinforcement deliveries. One way to address this issue is to evaluate the effects of DA depletions using interval schedules. Correa et al. (2002) compared the effects of accumbens DA depletions on a variable interval 30-s schedule (VI 30) and a tandem VI 30/FR 5 schedule (i.e. a VI 30 schedule with an increased ratio requirement added). DA depletions did not affect VI 30 instrumental responding (see also Sokolowski \& Salamone, 1998), but significantly decreased responding on the VI 30/FR 5 schedule. Thus, the ratio component of the schedule was demonstrated to be an important factor for determining sensitivity to DA depletion, while the temporal factor (i.e. intermittence), in the 30-s time range at least, did not seem to be a critical factor. The present study was designed to investigate further the effects of DA depletions on the performance of interval schedules with various ratio and time requirements. Correa et al. (2002) suggested that one of the effects of accumbens DA depletions may be to reduce the activating (i.e. ratefacilitating) effects of ratio requirements, but this needs to be investigated further by studying larger ratios (e.g. FR 10), and directly tested by providing a more detailed analysis of behavior (e.g. analysis of interresponse times). Another consideration is that Correa et al. (2003) used a 30-s time value for the VI schedule; this is a moderate length time interval, and it is important to explore the impact of longer intervals (e.g. 60 or $120 \mathrm{~s}$ ). Therefore, the present study examined the effects of accumbens DA depletions on four schedules: VI 60/FR 10, VI 60/FR 1, VI 120/FR 10 and VI 120/FR 1.

\section{Materials and methods}

\section{Experimental animals}

Male Sprague-Dawley rats (Harlan Sprague-Dawley, Indianapolis, IN, USA) weighing between 293 and $351 \mathrm{~g}$ at the beginning of the study $(n=96)$ were housed in a colony maintained at $23{ }^{\circ} \mathrm{C}$ with a 12-h light-dark cycle (lights on at 08:00 h). Rats were food restricted to $12 \mathrm{~g}$ /day for at least 8 days prior to training, such that they weighed approximately $96.3 \%( \pm 0.39)$ of their free-feeding body weights on the first day of training. Throughout the experiment, animals were given additional food (up to $12 \mathrm{~g}$ a day) and allowed modest growth. Water was available ad libitum in the home cages at all times. Animals were housed in pairs before surgery and placed in single cages afterwards. All experimental methods were performed in accordance with the Guide for the Care \& Use of Laboratory Animals, National Research Council, National Academy Press, 1996. The animal procedures were approved by the Institutional Animal Care and Use Committee of the University of Connecticut.

\section{Behavioral procedures and pre-surgery acquisition}

Experimental sessions were conducted in operant chambers $(30 \times 24 \times 21 \mathrm{~cm}$; Medical Associates, St. Albans, VT, USA $)$ that contained a lever and a food tray into which 45-mg Noyes Precision Food Pellets (Research Diets, New Brunswick, NJ, USA) were delivered. Animals were trained for 30-min sessions 5 days per week during the light part of the light-dark cycle for 6 weeks before surgery and 4 weeks after surgery. After 1 week of FR 1 training, rats were randomly assigned to four groups and trained on one of four different reinforcement schedules for 5 weeks: VI 60/FR 10, VI 60/FR 1, VI 120/FR 10, VI 120/FR 1. For the VI 60/FR 1 and VI 120/FR 1 schedules, rats were reinforced for the first response after each variable interval elapsed. Rats on VI 60/FR 10 and VI 120/FR 10 schedules were required to make ten responses after each interval elapsed to receive reinforcement. Each schedule contained seven different intervals that were derived from the formula developed by Catania \& Reynolds (1968). The seven intervals for the VI 60/FR 10 and VI 60/FR 1 schedules were 8.64, 18.73, 30.83, 45.95, 66.11, 96.36 and $156.36 \mathrm{~s}$. The seven intervals for the VI 120/FR 10 and VI 120/FR 1 schedules were 17.21, 38.04, 62.14, 92.27, 132.44, 192.69 and 313.19 s. Using a Medical-PC computer package (WMPC, Medical Associates), the program was configured such that an interval was randomly chosen without replacement from the set of seven intervals following each pellet delivery. This procedure ensured that animals experienced each interval once before experiencing it again.

Behavioral measures during the acquisition phase of this study included number of food pellets obtained, number of lever presses and interresponse times (IRTs, i.e. time between the onset of two consecutive responses). IRTs were assigned to 1-s bins, and the following time bins were recorded: bin 1, 0-1 s; bin 2, 1-2 s; bin 3, 2$3 \mathrm{~s}$; bin 4, 3-4 s; bin 5, 4-5 s; bin 6, 5-6 s; bin 7, 6-7 s; bin 8, 7-8 s; bin $9,8-9 \mathrm{~s}$; bin $10,9-10 \mathrm{~s}$; bin $11,10-11 \mathrm{~s}$; bin $12,11-12 \mathrm{~s}$; bin 13 , $12-13 \mathrm{~s}$; bin $14,13-14 \mathrm{~s}$; bin $15,14-15 \mathrm{~s}$; bin $16,15-16 \mathrm{~s}$; bin 17 , $16-17 \mathrm{~s}$; bin $18,17-18 \mathrm{~s}$; bin $19,18-19 \mathrm{~s}$; bin $20,19-20 \mathrm{~s}$; and bin $21,>20 \mathrm{~s}$, which was designated a pause.

\section{Surgery}

Assignment to treatment groups was counterbalanced for leverpressing performance prior to surgery. Prior to intra-accumbens injections of 6-hydroxydopamine (6-OHDA) or vehicle, rats were anesthetized with a solution $(1.0 \mathrm{~mL} / \mathrm{kg}$, i.p.) containing $10 \mathrm{~mL}$ ketamine $(100 \mathrm{mg} / \mathrm{mL})$ and $0.75 \mathrm{~mL}$ xylazine $(20 \mathrm{mg} / \mathrm{mL}$; both from Phoenix Scientific, Inc., St. Joseph, MO, USA). The MAO inhibitor pargyline (20.0 mg/kg, i.p.; Sigma-Aldrich Co., St. Louis, MO, USA) was injected $30 \mathrm{~min}$ prior to surgery to potentiate the effects of the neurotoxic agent. During surgery, rats received bilateral injections of either 6-OHDA hydrobromide $(n=60)$ or L-ascorbic acid $(0.1 \%$; $n=36$; both from Sigma-Aldrich). To obtain lesions, $12.5 \mu \mathrm{g}$ 6-OHDA dissolved in $1.5 \mu \mathrm{L}$ ascorbic acid was injected per side (1.5 $\mu \mathrm{L}$ of $8.33 \mu \mathrm{g} / \mu \mathrm{L}$ 6-OHDA). Control injections consisted of $1.5 \mu \mathrm{L}$ of $0.1 \%$ ascorbic acid per side. All injections were performed via 30-gauge stainless steel injectors placed directly into the nucleus accumbens $(\mathrm{AP}+2.8 \mathrm{~mm}, \mathrm{ML} \pm 1.4 \mathrm{~mm}, \mathrm{DV}-7.8 \mathrm{~mm}$; incisor bar $5.0 \mathrm{~mm}$ above the interaural line). Injections occurred at a rate of $0.3 \mu \mathrm{L} / \mathrm{min}$ for $5 \mathrm{~min}$ and were driven by a Harvard apparatus syringe pump. Following injections, injectors were left in place for $2 \mathrm{~min}$ to restrict diffusion.

\section{Effects of DA depletion}

After 3 days of post-surgery recovery, rats were tested during 30-min sessions 5 days/week for 4 weeks on their respective pre-surgery schedules: experiment 1, VI 60/FR 10; experiment 2, VI 60/FR 1; experiment 3, VI 120/FR 10; experiment 4, VI 120/FR 1. Behavioral measures during this testing phase included number of food 
pellets obtained, number of lever presses and IRTs as described above.

\section{Neurochemical analyses for tissue DA}

After completion of behavioral testing, rats were exposed to carbon dioxide for $30 \mathrm{~s}$ and decapitated. Brains were quickly removed and frozen on a Leitz Wetzlar microtome. Coronal sections of $750 \mu \mathrm{m}$ were cut through the nucleus accumbens and ventrolateral striatum (VLS). A 16-gauge stainless steel tube was used to dissect bilateral cylindrical samples from the accumbens and VLS. These tissue samples were then placed in $200 \mu \mathrm{L}$ of $0.1 \mathrm{~N}$ perchloric acid, homogenized, centrifuged and frozen. The supernatant was subsequently analysed using high-performance liquid chromatography and electrochemical detection as described previously (Cousins et al., 1993; Correa et al., 2002).

\section{Statistical analyses}

Neurochemical data were assessed using factorial ANOVAs (treatment $\times$ schedule), which were conducted separately for nucleus accumbens and VLS tissue samples. VLS samples from four animals were excluded from final analyses owing to tissue damage during sample extraction. Only data collected from 6-OHDA-treated rats with accumbens DA depletions greater than $80 \%$ (i.e. DA levels $<20 \%$ of the control mean) and VLS DA levels greater than $60 \%$ of the control mean were included for statistical analyses. Sixteen 6-OHDA-treated animals (VI 60/FR 10, $n=5$; VI 60/FR 1, $n=3$; VI 120/FR 10, $n=3$; VI $120 /$ FR $1, n=5$;) were excluded because they did not meet these criteria. Final numbers for 6-OHDA-treated groups were as follows: VI 60/FR 10, $n=11$; VI 60/FR 1, $n=11$; VI 120/FR 10, $n=13$; VI 120/FR 1, $n=9$. Final numbers for ascorbic acid vehicle groups were as follows: VI 60/FR $10, n=9$; VI 60/FR $1, n=9$; VI $120 /$ FR $10, n=9$; VI $120 /$ FR $1, n=8$.

Prior to data analyses, we calculated weekly averages for number of lever presses emitted and number of food pellets obtained. IRT bin data were also analysed after computing weekly averages of the absolute values for each bin. To analyse pre-surgery acquisition data (i.e. lever presses, food pellets), we used a 2 (VI schedule) $\times 2$ (FR schedule) $\times 5$ (week) factorial ANOVA with repeated measures on the week factor. To investigate the effects of adding a ratio component to the VI schedules on IRT distributions, separate ANOVAs were conducted for each group of two schedules featuring the same VI time component. More specifically, IRT data from the week immediately prior to surgery were analysed using a 2 (FR schedule) $\times 21$ (bin) factorial ANOVA, with repeated measures on the bin factor. Postsurgery data for total responses and food pellets obtained were analysed using a 2 (ratio requirement) $\times 2$ (interval level) $\times 2$ (DA depletion treatment) $\times 4$ (week) factorial ANOVA, with repeated measures on the week factor. Interaction comparisons and simple main effects using the error term from individual analyses were conducted to determine the sources of interaction effects (Keppel, 1991). For analysis of post-surgery IRT data in week 1 after surgery, two parameters were singled out for statistical analyses: fast responses (IRTs $<1.0 \mathrm{~s}$ ) and pauses (IRTs $>20.0 \mathrm{~s}$ ). These parameters were analysed using a 2 (ratio requirement) $\times 2$ (interval level) $\times 2$ (DA depletion treatment) factorial ANOVA. The use of these parameters was based upon an analysis of the overall IRT distributions shown during acquisition, and also upon previous research demonstrating that fast IRTs and pauses are the measures in the IRT distribution that are most sensitive to the effects of DA depletions and drugs injected into basal ganglia structures (Salamone et al., 1993a; Sokolowski \& Salamone, 1998; Correa et al., 2003). Significant interactions that were indicative of treatment-specific IRT effects were further analysed for simple effects using the error terms from the specific analyses. For all ANOVA calculations, a computerized statistical package (SPSS 10.1) was used $(\alpha=0.05)$. We also calculated the percentage of response reduction that was due to a reduction in fast responses [(mean IRTs bin 1 control - mean IRTs bin 1 DA depleted/mean responses control - mean responses DA depleted) $\times 100$ ].

\section{Results \\ Neurochemical results}

Table 1 gives the DA levels (ng/mg of tissue) in the nucleus accumbens and VLS for the four schedules following 4 weeks of testing post-surgery. Factorial ANOVA for DA levels in the nucleus accumbens revealed no significant main effect of schedule $\left(F_{3,71}=1.45, P=0.235\right)$ and no significant schedule-treatment interaction $\left(F_{3,71}=0.911, P=0.440\right)$. However, there was a significant main effect of treatment $\left(F_{1,71}=835.22, P<0.01\right)$, revealing that all 6-OHDA-treated animals had significantly less DA in the nucleus accumbens ( $>90 \%$ decrease), relative to vehicle controls. As expected, there were no significant differences in the data obtained from VLS samples (schedule, $F_{3,67}=0.654, P=0.583$; treatment, $F_{1,67}=0.719, \quad P=0.400 ; \quad$ schedule $\times$ treatment, $\quad F_{3,67}=0.476$, $P=0.700)$.

\section{Acquisition phase}

Data obtained during the pre-surgery training period were collapsed across post-acquisition treatment conditions for each schedule due to the absence of any significant pre-treatment-assignment-based differences. Figure 1 shows lever presses emitted during the 5 weeks of presurgery baseline training. As expected, a factorial ANOVA (VI schedule $\times$ FR schedule $\times$ week) demonstrated acquisition of lever pressing behavior by rats, regardless of schedule (main effect of week, $\left.F_{4,300}=189.157, P<0.01\right)$. There were also significant effects of VI schedule $\left(F_{1,75}=16.733, P<0.01\right)$ and FR schedule $\left(F_{1,75}=56.432, P<0.01\right)$, indicating that both interval and ratio components affected the rate of responding. Moreover, there were significant interactions between VI schedule and week and between FR schedule and week. Collapsed across FR schedules, rats on VI 60 schedules exhibited a greater increase in lever pressing during training, relative to rats on VI 120 schedules $\left(F_{4,300}=6.265, P<0.01\right)$.

TABLE 1. Neurochemical data

DA levels (ng/mg tissue)

\begin{tabular}{lrrrr}
\cline { 2 - 5 } Group & Nucleus accumbens & $(n)$ & \multicolumn{1}{l}{ VLS } & $(n)$ \\
\hline VI 60/FR 10 - 6-OHDA & $0.59 \pm 0.10$ & $(11)$ & $13.28 \pm 1.18$ & $(11)$ \\
VI 60/FR 10 - vehicle & $11.27 \pm 0.83$ & $(9)$ & $13.16 \pm 1.38$ & $(9)$ \\
VI 60/FR 1 - 6-OHDA & $0.82 \pm 0.20$ & $(11)$ & $14.04 \pm 1.19$ & $(11)$ \\
VI 60/FR 1 - vehicle & $12.72 \pm 0.72$ & $(9)$ & $14.33 \pm 0.53$ & $(8)$ \\
VI 120/FR 10 - 6-OHDA & $0.63 \pm 0.12$ & $(13)$ & $12.06 \pm 0.71$ & $(12)$ \\
VI 120/FR 10 - vehicle & $12.09 \pm 0.87$ & $(9)$ & $14.16 \pm 0.98$ & $(8)$ \\
VI 120 - 6-OHDA & $0.59 \pm 0.11$ & $(9)$ & $12.66 \pm 1.03$ & $(8)$ \\
VI 120 - vehicle & $10.85 \pm 0.96$ & $(8)$ & $12.89 \pm 0.80$ & $(8)$ \\
\hline
\end{tabular}

Values are mean ( \pm SEM) DA levels (ng/mg tissue) in nucleus accumbens or ventrolateral striatum (VLS) for the eight groups of rats on four different schedules following 4 weeks of post-surgery testing. $n=$ number of rats. 


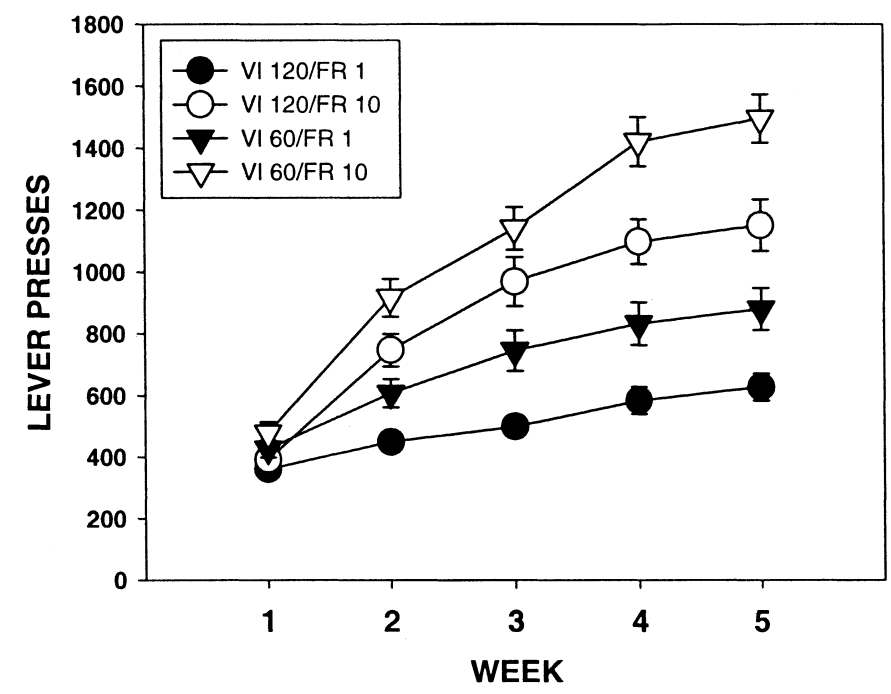

FIG. 1. Pre-surgery acquisition data. Mean $( \pm$ SEM) number of lever presses emitted by rats on four different schedules (VI 60/FR 10, VI 60/FR 1, VI 120 FR 10, VI 120/FR 1) during the 5-week acquisition phase.
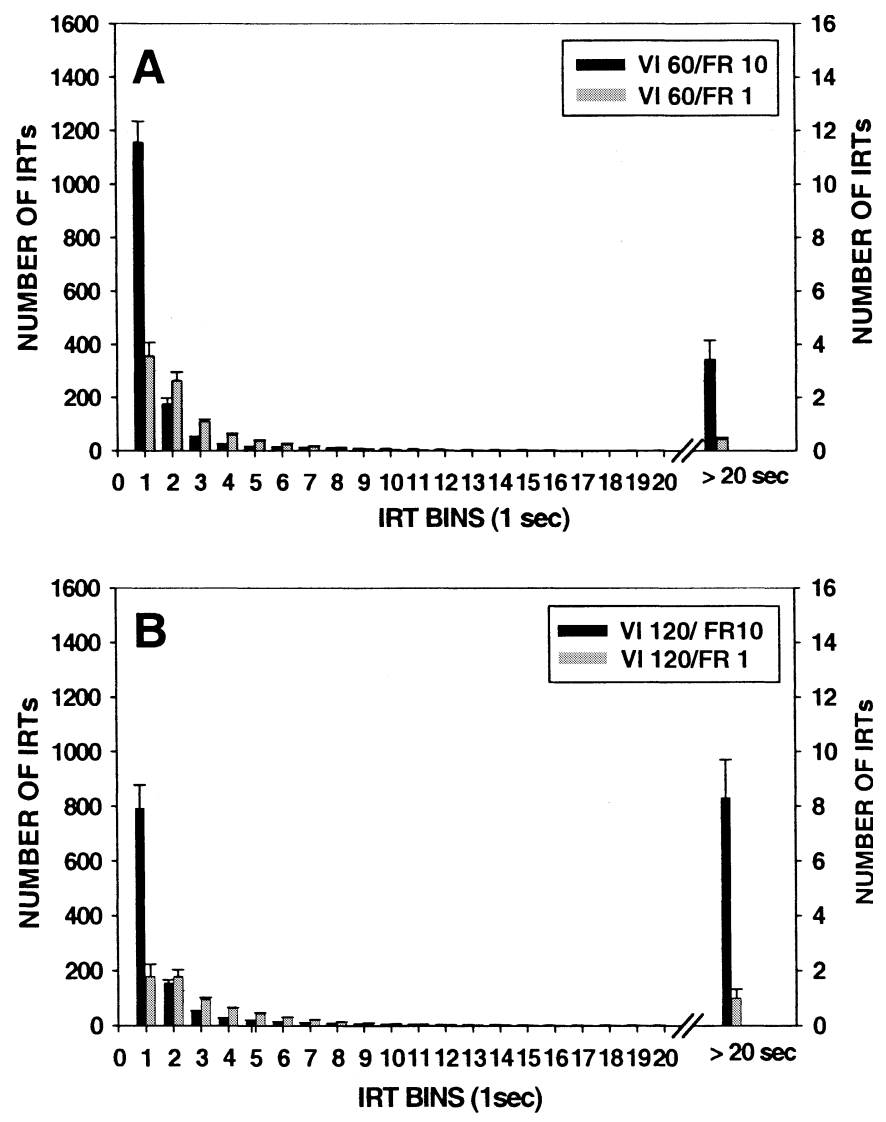

FIG. 2. Pre-surgery acquisition data. Distribution of interresponse times (IRTs) for each group of two schedules featuring the same VI time component during the week immediately prior to surgery. (A) VI 60/FR 1 and VI 60/FR 10 schedules. (B) VI 120/FR 1 and VI 120/FR 10 schedules.

Collapsed across VI schedules, rats on FR 10 schedules exhibited a greater increase in lever pressing during training, relative to rats on FR 1 schedules $\left(F_{4,300}=34.985, P<0.01\right)$. There was no significant three-way interaction $\left(\mathrm{VI} \times \mathrm{FR} \times\right.$ week, $\left.F_{1,75}=0.323, P=0.571\right)$.
IRT distributions for each schedule during the week immediately preceding surgery are illustrated in Fig. 2A and B. Repeated-measures ANOVA for both VI 60 schedules (i.e. VI 60 and VI 60/FR 10; Fig. 2A) revealed a significant main effect of FR schedule $\left(F_{1,38}=35.27, \quad P<0.01\right)$, a significant main effect of bin $\left(F_{20,760}=217.85, P<0.01\right)$, and a significant FR schedule $\times$ bin interaction $\left(F_{20,760}=61.86, P<0.01\right)$. The significant interaction reflected the different IRT distributions generated by the different ratio components (i.e. FR 1 and FR 10) attached to the VI 60 schedule. Repeated-measures ANOVA for the VI 120 schedules (i.e. VI 120 and VI 120/FR 10; Fig. 2B) revealed a significant main effect of FR schedule $\left(F_{1,37}=16.14, P<0.01\right)$, a significant main effect of bin $\left(F_{20,740}=78.58, P<0.01\right)$, and a significant FR schedule $\times$ bin interaction $\left(F_{20,740}=31.22, P<0.01\right)$. Again, the significant interaction reflected the different IRT distributions generated by the different ratio components (i.e. FR 1 and FR 10) attached to the VI 120 schedule. As shown in Fig. 2, a major effect of adding a ratio requirement across both VI levels was a dramatic increase in the number of fast IRTs (i.e. IRTs $<1.0 \mathrm{~s}$ ).

Table 2 shows food pellets obtained during the 5 weeks of presurgery baseline training. Statistical analysis revealed main effects of VI schedule, FR schedule, and week on food pellets obtained $\left(F_{1,75}=5042.21, \quad F_{1,75}=563.21, \quad F_{4,300}=322.61\right.$, respectively, $P<0.01)$. VI $\times$ week and FR $\times$ week interactions were also present $\left(F_{4,300}=66.84, F_{4,300}=98.60\right.$, respectively, $\left.P<0.01\right)$. In addition, there was a two-way interaction between VI schedule and FR schedule $\left(F_{1,75}=79.86, P<0.01\right)$ and a three-way interaction between VI schedule, FR schedule and week $\left(F_{4,300}=21.11, P<0.01\right)$. These interactions reflect the fact that rats on schedules with the shorter interval component (VI 60/FR 10, VI 60/FR 1) received more pellets per session than did rats on schedules with the longer interval component (VI 120/FR 10, VI 120/FR 1).

\section{Effects of $D A$ depletions}

Figure $3 \mathrm{~A}$ and $\mathrm{B}$ depicts lever pressing data for DA-depleted and vehicle-treated rats responding on all four schedules (Fig. 3A, VI 60/FR 10 and VI 60/FR 1 schedules; Fig. 3B, VI 120/FR 10 and VI 120/FR 1 schedules) during the 4-week post-surgery testing phase. Four-way ANOVA revealed that there was an overall effect of FR requirement (i.e. 1 vs. $10 ; F_{1,71}=10.8, P<0.001$ ), and an overall effect of VI level (i.e. $60 \mathrm{~s}$ vs. $120 \mathrm{~s} ; F_{1,71}=48.5, P<0.001$ ), but no significant overall effect of DA depletion treatment $\left(F_{1,71}=0.96\right.$, $P>0.3)$. None of the other interactions that were solely dealing with between-groups variables was significant. There was an overall significant effect of week $\left(F_{3,213}=36.2, P<0.001\right)$. In addition, there was a significant week $\times$ FR level interaction $\left(F_{3,213}=8.3\right.$, $P<0.001)$ and a significant DA depletion $\times$ week $\times$ FR requirement interaction $\left(F_{3,213}=4.4, P<0.01\right)$. None of the interactions involving the VI levels was statistically significant. These analyses indicate that there was an effect of DA depletion that depended upon the week and the FR requirement, but not on the interval level. Additional analyses were undertaken to identify the source of the interaction involving the DA depletion effect. Separate analyses for each week indicated that there was only a significant DA depletion $\times$ FR requirement interaction during the first week after surgery $\left(F_{1,75}=6.9, P<0.01\right)$. Further analyses of the week 1 data demonstrated that there was a significant effect of DA depletion among those rats performing on the two schedules with FR 10 components (VI 60/FR 10 and VI 120/FR 10; $\quad F_{1,42}=19.1$, $P<0.001$ ), but not for the rats performing on the schedules with 
TABLE 2. Number of pellets obtained by rats on four different schedules during the acquisition phase

\begin{tabular}{lrrrr}
\hline Schedule & Week 1 & Week 2 & Week 3 & Week 4 \\
\hline VI 60/FR 10 & $16.13 \pm 0.56$ & $22.38 \pm 0.45$ & $23.72 \pm 0.28$ & $24.69 \pm 0.25$ \\
VI 60/FR 1 & $26.20 \pm 0.22$ & $28.13 \pm 0.13$ & $28.45 \pm 0.13$ & $28.57 \pm 0.10$ \\
VI 120/FR 10 & $9.28 \pm 0.27$ & $11.55 \pm 0.17$ & $12.25 \pm 0.15$ & $12.46 \pm 0.12$ \\
VI 120/FR 1 & $13.48 \pm 0.15$ & $14.22 \pm 0.12$ & $14.24 \pm 0.07$ & $14.38 \pm 0.05$ \\
\hline
\end{tabular}

Data are presented as means $\pm \mathrm{SEM}$.
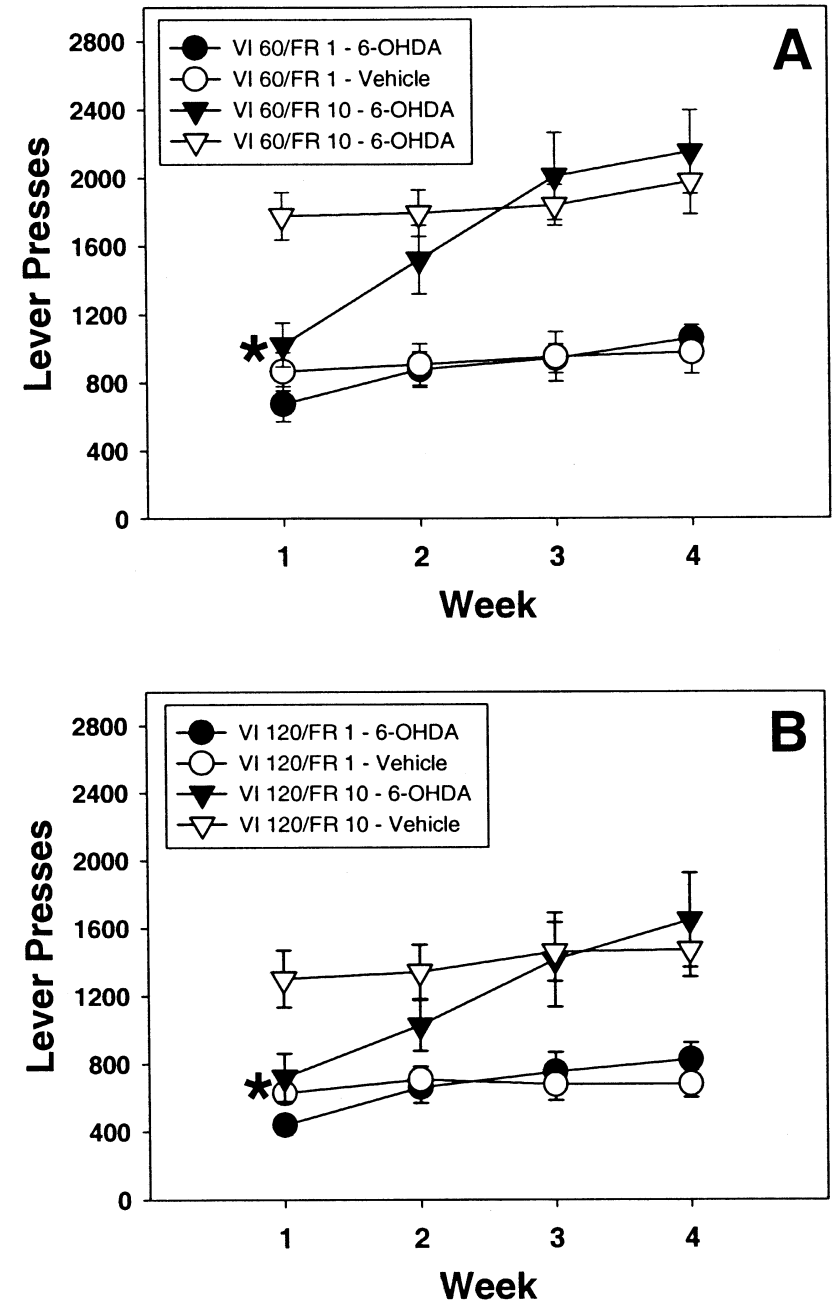

FIG. 3. Mean ( \pm SEM) number of lever presses emitted by vehicle- and 6-OHDA-treated rats during the test phase following surgery. (A) VI 60/ FR 1 and VI 60/FR 10 schedules. (B) VI 120/FR 1 and VI 120/FR 10 schedules. ${ }^{*} P<0.05$, significantly different from vehicle. the FR 1 components (VI 60/FR 1 and VI 120/FR $1 ; F_{1,42}=3.6$, $P=0.066$ ). Table 3 shows the number of pellets received by 6-OHDA- and vehicle-treated rats performing on all four schedules. As was the case with the analysis of lever presses, there was a significant DA depletion $\times$ week $\times$ FR level interaction for the number of pellets $\left(F_{3,213}=5.1, P<0.002\right)$.

Figure $4 \mathrm{~A}$ and $\mathrm{B}$ shows the number of fast responses (i.e. IRTs $<1.0 \mathrm{~s}$ ) and pauses (i.e. IRTs $>20.0 \mathrm{~s}$ ) for 6-OHDA- and vehicletreated rats across all four schedules during the first week post-surgery. For fast responses (Fig. 4A) there was a significant overall DA depletion treatment effect $\left(F_{1,71}=18.6, P<0.001\right)$, a significant overall effect of VI level $\left(F_{1,71}=11.0, P<0.001\right)$ and a significant effect of FR requirement $\left(F_{1,71}=82.2, P<0.0 .001\right)$. There was no significant DA depletion $\times$ VI level interaction $\left(F_{1,71}=0.7\right.$, n.s. $)$, but there was a significant DA depletion $\times$ FR requirement interaction $\left(F_{1,71}=9.7 .0, P<0.01\right)$. This demonstrates that the effect of DA depletion depended upon the FR requirement. Analysis of the data from the animals that had an added FR 10 requirement (collapsed across VI levels) showed that there was a significant DA depletion effect $\left(F_{1,40}=16.6, P<0.001\right)$. However, there was no DA depletion effect in the animals responding on the two VI schedules with only the FR 1 requirement $\left(F_{1,40}=2.2\right.$, n.s. $)$. The percentage of total response reduction due to reduction in fast responses (i.e. bin 1 , IRT $<1.0 \mathrm{~s}$ ) was calculated for each of the two schedules with added FR 10 requirements, because these schedules showed significant DA depletion effects on fast responses as well as total responding. Ninety-one per cent of the total reduction in responding produced by DA depletion in the VI 60/FR 10 schedule and $85 \%$ of the total reduction in responding produced by DA depletion in the VI 120/FR 10 schedule were due to reductions in fast responses. Analyses of the number of pauses (IRTs $>20.0 \mathrm{~s}$; Fig. 4B) revealed that there was a significant overall DA depletion effect $\left(F_{1,71}=35.16\right.$, $P<0.001)$, a significant overall effect of VI level $\left(F_{1,71}=5.8\right.$, $P<0.05)$ and a significant effect of FR requirement $\left(F_{1,71}=27.2\right.$, $P<0.0 .001)$. There was no significant DA depletion $\times$ VI level interaction $\left(F_{1,71}=0.2\right.$, n.s. $)$, and unlike the analysis of fast IRTs, the DA depletion $\times$ FR requirement interaction was not statistically significant $\left(F_{1,71}=3.7, P=0.06\right)$. This demonstrates that there was an overall effect of DA depletion on number of pauses, and this effect

TABLE 3. Effects of accumbens DA depletions on number of pellets obtained by rats on four different schedules during the testing phase (mean \pm SEM)

\begin{tabular}{llrr}
\hline Group & \multicolumn{1}{l}{ Week 1 } & \multicolumn{1}{l}{ Week 2 } & Week 3 \\
\hline VI 60/FR 10 - 6-OHDA & $21.38 \pm 1.13^{*}$ & $24.0 \pm 1.04$ & $25.29 \pm 0.62$ \\
VI 60/FR 10 - vehicle & $25.09 \pm 0.36$ & $25.44 \pm 0.31$ & $25.76 \pm 0.26$ \\
VI 60/FR 1 - 6-OHDA & $27.29 \pm 0.68$ & $28.24 \pm 0.39$ & $28.65 \pm 0.28$ \\
VI 60/FR 1 - vehicle & $28.33 \pm 0.17$ & $28.53 \pm 0.13$ & $28.62 \pm 0.22$ \\
VI 120/FR 10 - 6-OHDA & $10.35 \pm 0.63^{*}$ & $11.82 \pm 0.42$ & $12.49 \pm 0.34$ \\
VI 120/FR 10 - vehicle & $12.51 \pm 0.30$ & $12.82 \pm 0.18$ & $12.98 \pm 0.19$ \\
VI 120/FR 1 - 6-OHDA & $13.76 \pm 0.24$ & $14.27 \pm 0.17$ & $14.24 \pm 0.12$ \\
VI 120/FR 1 - vehicle & $14.30 \pm 0.10$ & $14.45 \pm 0.05$ & 14.14 \\
& & & $14.53 \pm 0.15$ \\
\hline
\end{tabular}

Data are presented as means \pm SEM. $* P<0.05$, significantly different from vehicle. 

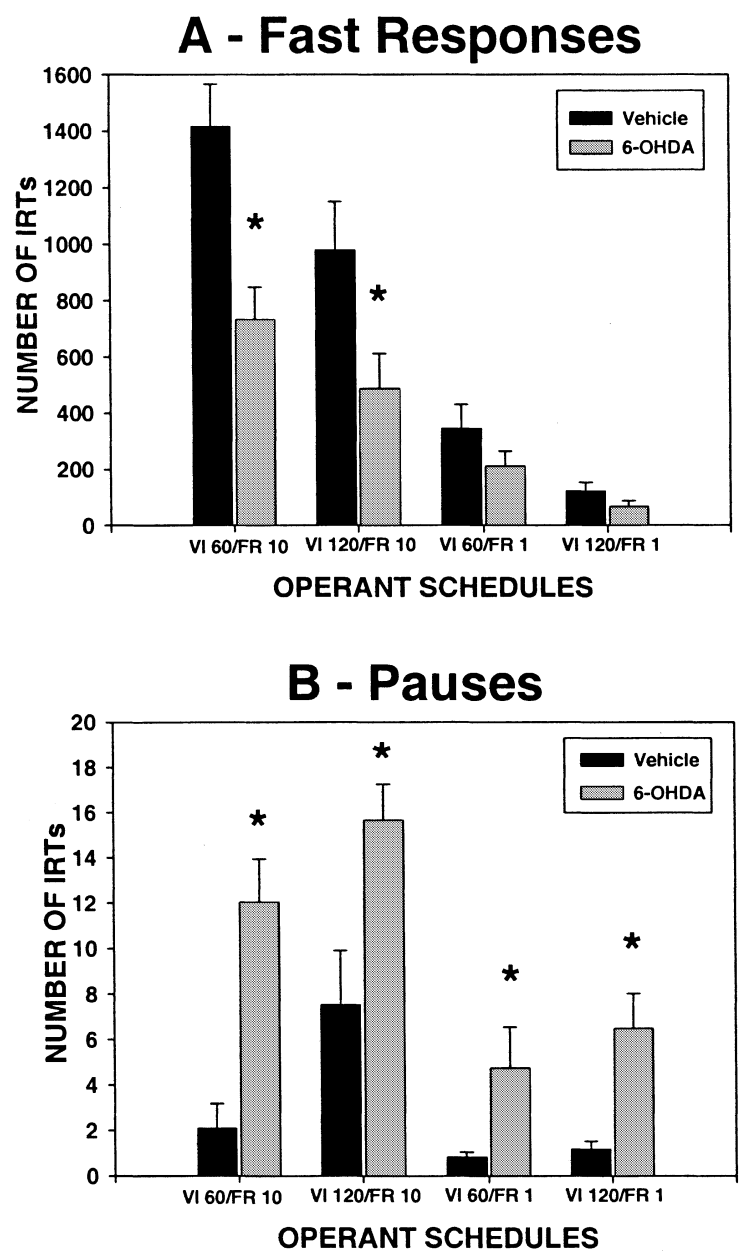

FIG. 4. Parameters obtained from analyses of interresponse time (IRT; time between the onset of two consecutive responses) data during the 1st week after surgery. (A) Number of fast responses (i.e. IRTs $<1.0 \mathrm{~s}$ ) (B) Number of pauses (i.e. IRTs $>20.0 \mathrm{~s}$ ). ${ }^{*} P<0.05$, significantly different from vehicle.

did not depend upon the FR requirement. Because the DA depletion $\times$ FR requirement interaction approached significance, additional analyses were performed, which showed that DA depletion effects were significant under both FR 10 and FR 1 conditions when the data were collapsed across VI levels (FR 10, $F_{1,40}=23.1$, $P<0.001$; FR $\left.1, F_{1,35}=12.2, P<0.001\right)$.

\section{Discussion}

\section{Summary of results}

As depicted in Figs 1 and 2, attachment of the additional ratio requirement to the interval schedules led to greater response rates. Animals on both VI 60/FR 10 and VI 120/FR 10 schedules emitted more fast responses (i.e. had an increased number of IRT bins $<1 \mathrm{~s}$ ) relative to animals responding on the corresponding VI/FR 1 schedules. Nucleus accumbens DA depletions significantly impaired responding of rats performing on the schedules with the higher ratio requirement (i.e. VI 60/FR 10, VI 120/FR 10). DA-depleted rats responding on the schedules with added FR 10 components showed significant decreases in fast responses and significant increases in the number of pauses. Overall responding on the schedules with FR 1 components (i.e. VI 60/FR 1 and VI 120/FR 1) was not substantially affected by accumbens DA depletion, but the number of pauses was increased for rats responding on these schedules. The response-suppressing effects of DA depletions were significant only during the first week post-surgery. Thus, DA-depleted rats showed recovery of function over succeeding weeks, which is consistent with previous studies (Zigmond et al., 1984; Wolterink et al., 1990; Salamone et al., 1993a; Correa et al., 2002; Ishiwari et al., 2004).

\section{Effects of DA depletions on ratio and interval performance}

Accumbens DA depletions suppressed operant responding on both VI 60/FR 10 and VI 120/FR 10 schedules, which is consistent with previous results indicating that schedules with ratio requirements are sensitive to the effects of accumbens DA depletions (Aberman \& Salamone, 1999; Salamone et al., 2001; Correa et al., 2002; Salamone $\&$ Correa, 2002). The overall interaction between ratio requirement and DA depletion in week 1 and the significant DA depletion effects in rats that had the FR 10 requirement attached to the interval attest to the substantial role that ratio requirements play in making operant schedules sensitive to the effects of DA depletion. The reductions in responding on VI 60/FR 10 and VI 120/FR 10 schedules that were produced by DA depletions were accompanied by substantial changes in IRT parameters (i.e. reductions in the number of fast responses, increases in pausing). In particular, the effects of DA depletion on VI 60/FR 10 and VI 120/FR 10 performance appear to be largely dependent on a reduction in the number of fast responses (i.e. $<1.0 \mathrm{~s}$, or bin 1). Schedules with FR 10 components had a large number of fast responses, and $85-91 \%$ of the total reductions in responding produced by DA depletions on these schedules were due to reductions in fast responses. Taken together, these findings demonstrate that accumbens DA depletions have substantial effects on schedules with moderate ratio requirements, and that a predominant aspect of these effects is a decrease in the behavioral activation produced by attaching a ratio requirement. For rats tested on the schedules with added FR 10 components, DA depletion suppressed responding in week 1 postsurgery to a level that was comparable with that shown by control rats responding on the VI schedules without the added ratio. Thus, attachment of a ratio requirement to an interval schedule presents a challenge to the animal, and rats adapt to this obstacle by increasing response rates. The ability of rats to adapt to the challenge presented by the ratio requirement appears to be dependent upon the integrity of the DA innervation of the nucleus accumbens.

Interval requirements appeared to play a less robust role than ratio requirements in determining the schedule-dependent effects of DA depletion. Although the two VI levels used in the present study did generate different rates, as indicated by the significant main effects of VI level shown across several analyses, interval level did not interact with DA depletion in any of the analyses performed. The overall effect of DA depletion collapsed across the two schedules with FR 1 components approached but did not reach statistical significance. There was a tendency for DA-depleted rats in the VI 120 schedule to show slight reductions in responding, and further research should explore the use of longer VI intervals. Nevertheless, the present results are consistent with previous studies using the VI 30 schedule (Sokolowski \& Salamone, 1998; Correa et al., 2002). Together with previous reports, the present results support the observation that the effects of accumbens DA depletion are ratio-dependent, and suggest that intermittence over a time range up to $120 \mathrm{~s}$ does not substantially increase sensitivity to the effects of accumbens DA depletions. In contrast to the schedules with FR 10 components, the results obtained with the VI 60 or VI 120/FR 1 schedules showed that DA depletion 
did not significantly reduce the number of fast responses. Thus, on several measures of behavior, the VI schedules with low ratio requirements were relatively insensitive to the effects of accumbens DA depletions. Several factors could contribute to the apparent insensitivity of VI schedules to accumbens DA depletions. The temporal uncertainty produced by the variability of the time intervals in a VI schedule may be a factor, because fixed interval schedules with comparable time values (i.e. FI $30 \mathrm{~s}$ and FI $60 \mathrm{~s}$ ) have been shown to be impaired by accumbens DA depletions (Robbins et al., 1983; Cousins et al., 1999). Another factor could be that these schedules induce a slow local rate of responding (Salamone et al., 1999), and in the present studies the VI 60 and VI 120/FR 1 schedules did generate low overall rates of responding and a relatively flat IRT distribution. Nevertheless, the present results also suggest that low baseline response rates, per se, do not necessarily make animals completely insensitive to all the effects of accumbens DA depletions. Detailed analyses of the response patterns shown by DA-depleted animals responding on the VI 60/FR 1 and VI 120/FR 1 schedules revealed that there was a significant increase in the tendency to pause for long periods of time (i.e. IRTs $>20 \mathrm{~s}$ ). Thus, it appears that at the time intervals used in the present study (i.e. $60-120 \mathrm{~s}$ ), VI schedules can be sensitive to DA depletions, at least in terms of the tendency to take pauses in responding. The increased pausing produced by DA depletions across all four schedules may reflect a fragmentation of the pattern of responding, a lack of behavioral activation, reduced responsiveness to conditioned cues or problems initiating instrumental activity. Nevertheless, the specific characteristics of the effects of accumbens DA depletions on schedules with ratio components (i.e. VI 60 and VI 120/FR 10) clearly are different to the effects of those depletions on the performance of schedules with long intervals but minimal ratio requirements (e.g. VI 60 and VI 120/FR 1).

Previous research has focused on the role of time as a factor in determining sensitivity to dopaminergic manipulations or accumbens cell body lesions. Salamone et al. (2001) studied the effects of DA depletions on a variety of schedules that had the same programmed molar reinforcement density, but different organization of the response requirement (i.e. FR 50 for one food pellet, FR 100 for two pellets, FR 200 for four pellets, FR 300 for six pellets). DA depletions produced a slowing of response rate on the FR 50 schedule, but severely impaired responding on schedules with very large ratios (e.g. FR 200, FR 300), even though the programmed density of reinforcement and baseline response rates were similar across these different schedules. This led to the suggestion that DA depletions made animals more dependent upon the direct feedback provided by primary reinforcement, and that the temporal organization of the response requirement (i.e. the need to respond over long periods of time without primary reinforcement) was an important determinant of sensitivity to the effects of DA depletion (Salamone et al., 2001). Using a reinforcement-delay choice procedure, Cardinal et al. (2001) showed that excitotoxic lesions of the nucleus accumbens affected the tendency of rats to choose large delayed reinforcement over immediate small reinforcement. Dopaminergic drugs have been shown to alter the process of delay discounting (Richards et al., 1999). Nevertheless, although time may be a factor in tests related to preference, it is not clear that time requirements alone are a major determinant of sensitivity to the response-suppressing effects of accumbens DA depletions. For example, Wakabayashi et al. (2004) studied the effects of intra-accumbens injections of DA antagonists on a novel progressive delay task, and observed that blockade of D1 or D2 family receptors did not impair the ability of the rats to wait for sucrose reward. In addition, there is no evidence that the time intervals imposed by schedules with moderate ratio requirements are the primary explanation for the rate-suppressing effects of DA depletions on those schedules. Salamone et al. (2001) studied schedules with moderate-to-high ratio requirements (e.g. FR 20, FR 50), and observed that DA-depleted animals received food on average every $30 \mathrm{~s}$ for the FR 20 schedule and $56 \mathrm{~s}$ for the FR 50 schedule. Performance on these two schedules was disrupted by DA depletions. However, performance on interval schedules with similar levels of intermittence in reinforcement (e.g. VI 30, VI 60) was not significantly affected by accumbens DA depletions (present study; Sokolowski \& Salamone, 1998; Correa et al., 2002). An even more extreme example is the FR 5 schedule, which has been shown to be sensitive to the effects of accumbens DA depletions (Salamone et al., 1993a, 2001; Sokolowski \& Salamone, 1998; Ishiwari et al., 2004). DAdepleted animals responding on this schedule received reinforcement at an average rate of approximately one pellet every $8 \mathrm{~s}$ (Ishiwari et al., 2004), and yet there was still a deficit in response rate under these conditions. Thus, although we have evidence that VI schedules can make animals sensitive to the pause-enhancing effects of DA depletions, there is no evidence that the time intervals imposed by FR 5, FR 16, FR 20 and FR 50 schedules, in and of themselves, can explain the sensitivity to DA depletion that these ratio schedules engender.

\section{Conclusions}

Accumbens DA depletions produce schedule-dependent effects on operant responding, and the present studies have identified two distinct effects of DA depletion. Accumbens DA depletions suppress the tendency to emit high local rates of responding, and this effect results in a substantial reduction of responding on schedules with a ratio component added (i.e. VI 60 and VI 120/FR 10). The reduction of responding on the VI 60/FR 10 and VI 120/FR 10 schedules was not simply dependent upon the degree of intermittence in the schedule, because VI/FR 1 schedules with comparable time requirements were only minimally affected by DA depletion. In addition, DA depletions increased the tendency to take long pauses in responding (i.e. IRTs $>20.0 \mathrm{~s}$ ), and this effect was evident across all four schedules tested. Accumbens DA appears to play an important role in overcoming the work-related challenges presented by ratio schedules. This function is important for aspects of natural motivation and drug seeking behavior, and may be related to conditions such as psychomotor slowing in depression (Salamone et al., 2003).

\section{Acknowledgements}

This work was supported by a grant to J.D.S. from the US National Science Foundation and by a grant to S.M. from the Fundação para a Ciência e Tecnologia (Portugal).

\section{Abbreviations}

6-OHDA, 6-hydroxydopamine; DA, dopamine; FI, fixed interval; FR, fixed ratio; IRT, interresponse time; VI, variable interval; VLS, ventrolateral striatum.

\section{References}

Aberman, J.E. \& Salamone, J.D. (1999) Nucleus accumbens dopamine depletions make rats more sensitive to high ratio requirements but do not impair primary food reinforcement. Neuroscience, 92, 545-552.

Aberman, J.E., Ward, S.J. \& Salamone, J.D. (1998) Effects of dopamine antagonist and accumbens dopamine depletions on time-constrained progressive-ratio performance. Pharmacol. Biochem. Behav., 61, 341-348. 
Baldo, B.A., Sadeghian, K., Basso, A.M. \& Kelley, A.E. (2002) Effects of selective dopamine D1 or D2 receptor blockade within nucleus accumbens subregions on ingestive behavior and associated motor activity. Behav. Brain. Res., 137, 165-177.

Bassareo, V. \& DiChiara, G. (1999) Modulation of feeding-induced activation of mesolimbic dopamine transmission by appetitive stimuli and its relation to motivational state. Eur. J. Neurosci., 11, 4389-4397.

Berridge, K.C. (2000) Measuring hedonic impact in animals and infants: microstructure of affective taste reactivity patterns. Neurosci. Biobehav. Rev., 24, 173-198.

Berridge, K.C. \& Robinson, T.E. (1998) What is the role of dopamine in reward: hedonic impact, reward learning, or incentive salience? Brain Res. Rev., 28, 309-369.

Bickel, W.K., Marsch, L.A. \& Carroll, M.E. (2000) Deconstructing relative reinforcing efficacy and situating the measures of pharmacological reinforcement with behavioral economics: a theoretical proposal. Psychopharmacology, 153, 44-56.

Cador, M., Taylor, J.R. \& Robbins, T.W. (1991) Potentiation of the effects of reward-related stimuli by dopaminergic-dependent mechanisms in the nucleus accumbens. Psychopharmacology (Berlin), 104, 377-385.

Cardinal, R.N. \& Everitt, B.J. (2004) Neural and psychological mechanisms underlying appetitive learning: links to drug addiction. Curr. Opin. Neurobiol., 14, 156-162.

Cardinal, R.N., Parkinson, J.A., Hall, J. \& Everitt, B.J. (2002) Emotion and motivation: the role of the amygdala, ventral striatum, and prefrontal cortex. Neurosci. Biobehav. Rev., 26, 321-352.

Cardinal, R.N., Pennicott, D.R., Sugathapala, C.L., Robbins, T.W. \& Everitt, B.J. (2001) Impulsive choice induced in rats by lesions in the nucleus accumbens core. Science, 292, 2499-2501.

Carelli, R.M. (2002) Nucleus accumbens cell firing during goal-directed behaviors for cocaine vs 'natural' reinforcement. Physiol. Behav., 76, 379 387.

Catania, A.C. \& Reynolds, G.S. (1968) A quantitative analysis of the responding maintained by interval schedules of reinforcement. J. Exp. Anal. Behav., 11, 327-383.

Collier, G.H. \& Jenkins, W. (1969) Work as a determinant of instrumental performance. J. Compar. Physiol. Psychol., 68, 659-662.

Correa, M., Carlson, B.B., Wisniecki, A. \& Salamone, J.D. (2002) Nucleus accumbens dopamine and work requirements on interval schedules. Behav. Brain Res., 137, 179-187.

Correa, M., Mingote, S., Betz, A., Wisniecki, A. \& Salamone, J.D. (2003) Substantia nigra pars reticulata GABA is involved in the regulation of operant lever pressing: pharmacological and microdialysis studies. $\mathrm{Neu}$ roscience, 119, 759-766.

Cousins, M.S., Atherton, A., Turner, L. \& Salamone, J.D. (1996) Nucleus accumbens dopamine depletions alter relative response allocation in a T-maze cost/benefit task. Behav. Brain Res., 74, 189-197.

Cousins, M.S. \& Salamone, J.D. (1994) Nucleus accumbens dopamine depletions in rats affect relative response allocation in a novel cost/benefit procedure. Pharmacol. Biochem. Behav., 49, 85-91.

Cousins, M.S., Sokolowski, J.D. \& Salamone, J.D. (1993) Different effects of nucleus accumbens and ventrolateral striatal dopamine depletions on instrumental response selection in the rat. Pharmacol. Biochem. Behav, 44, 943-951.

Cousins, M.S., Trevitt, J., Atherton, A. \& Salamone, J.D. (1999) Different behavioral functions of dopamine in nucleus accumbens and ventrolateral striatum: a microdialysis and behavioral investigation. Neuroscience, 91, 925-934.

Datla, K.P., Ahier, R.G., Young, A.M., Gray, J.A. \& Joseph, M.H. (2002) Conditioned appetitive stimulus increases extracellular dopamine in the nucleus accumbens of the rat. Eur. J. Neurosci., 16, 1987-1993.

De Borchgrave, R., Rawlins, J.N.P., Dickinson, A. \& Balleine, B.W. (2002) Effects of cytotoxic nucleus accumbens lesions on instrumental conditioning rats. Exp. Brain Res., 144, 50-68.

Di Chiara, G. (2002) Nucleus accumbens shell and core dopamine: differential role in behavior and addiction. Behav. Brain Res., 137, 75-114.

Dickinson, A. \& Balleine, B. (1994) Motivational control of goal-directed action. Anim. Learn. Behav., 22, 1-18.

Everitt, B.J., Cador, M. \& Robbins, T.W. (1989) Interactions between the amygdala and ventral striatum in stimulus-reward associations: studies using a second-order schedule of sexual reinforcement. Neuroscience, 30, 63-75.

Everitt, B.J., Parkinson, J.A., Olmstead, M.C., Arroyo, M., Robledo, P. \& Robbins, T.W. (1999) Associative processes in addiction and reward. The role of amygdala-ventral striatal subsystems. Ann. NY Acad. Sci., 877, 412438.
Hamill, S., Trevitt, J.T., Nowend, K.L., Carlson, B.B. \& Salamone, J.D. (1999) Nucleus accumbens dopamine depletions and time-constrained progressive ratio performance: effects of different ratio requirements. Pharmacol. Biochem. Behav., 64, 21-27.

Horvitz, J.C. (2002) Dopamine gating of glutamatergic sensorimotor and incentive motivational input signals to the striatum. Behav. Brain Res., 137, 65-74.

Hursh, S.R., Raslear, T.G., Shurtleff, D., Bauman, R. \& Simmons, L. (1988) A cost-benefit analysis of demand for food. J. Exp. Anal. Behav., 50, 419-440.

Ikemoto, S. \& Panksepp, J. (1999) The role of nucleus accumbens dopamine in motivated behavior: a unifying interpretation with special reference to reward seeking. Brain Res. Rev., 31, 6-41.

Ishiwari, K., Weber, S., Mingote, S., Correa, M. \& Salamone, J.D. (2004) Accumbens dopamine and the regulation of effort in food seeking behavior: modulation of work output by different ratio or force requirements. Behav. Brain Res., 151, 83-91.

Kelley, A.E. (1999) Neural integrative studies of nucleus accumbens subregions in relation to learning and motivation. Psychobiology, 27, 198213.

Keppel, G. (1991) Design and Analysis: a Researchers Handbook. Prentice Hall, Englewood Cliffs, NJ.

Koch, M., Schmid, A. \& Schnitzler, H.U. (2000) Role of nucleus accumbens dopamine D1 and D2 receptors in instrumental and Pavlovian paradigms of conditioned reward. Psychopharmacology, 152, 67-73.

Koob, G.F., Riley, S.J., Smith, S.C. \& Robbins, T.W. (1978) Effects of 6-hydroxydopamine lesions of the nucleus accumbens septi and olfactory tubercle on feeding, locomotor activity, and amphetamine anorexia in the rat. J. Comp. Physiol. Psychol., 92, 917-927.

McCullough, L.S., Cousins, M.S. \& Salamone, J.D. (1993) The role of nucleus accumbens dopamine in responding on a continuous reinforcement schedule: a neurochemical and behavioral study. Pharmacol. Biochem. Behav., 46, 581-586.

Nicola, S.M., Yun, I.A., Wakabayashi, K.T. \& Fields, H.L. (2004) Cue-evoked firing of the nucleus accumbens neurons encodes motivational significance during a discriminative stimulus task. J. Neurophysiol., 91, 1840-1865.

Nowend, K.L., Arrizi, M., Carlson, B.B. \& Salamone, J.D. (2001) D1 or D2 antagonism in nucleus accumbens core or dorsomedial shell suppresses lever pressing for food but leads to compensatory increases in chow consumption. Pharmacol. Biochem. Behav., 69, 373-382.

Parkinson, J.A., Dalley, J.W., Cardinal, R.N., Bamford, A., Fehnert, B., Lachenal, G., Rudarakanchana, N., Halkerston, K.M., Robbins, T.W. \& Everitt, B.J. (2002) Nucleus accumbens dopamine depletion impairs both acquisition and performance of appetitive Pavlovian approach behaviour: implications for mesoaccumbens dopamine function. Behav. Brain Res., 137, 149-163.

Parkinson, J.A., Olmstead, M.C., Burns, L.H., Robbins, T.W. \& Everitt, B.J. (1999) Dissociation in effects of lesions of the nucleus accumbens core and shell on appetitive Pavlovian approach behavior and the potentiation of conditioned reinforcement and locomotor activity by D-amphetamine. J. Neurosci., 19, 2401-2411.

Richards, J.B., Sabol, K.E. \& de Wit, H. (1999) Effects of methamphetamine on the adjusting amount procedure, a model of impulsive behavior in rats. Psychopharmacology, 146, 432-439.

Robbins, T.W., Roberts, D.C. \& Koob, G.F. (1983) Effects of D-amphetamine and apomorphine upon operant behavior and schedule-induced licking in rats with 6-hydroxydopamine-induced lesions of the nucleus accumbens. J. Pharmacol. Exp. Ther, 224, 662-673.

Roitman, M.F., Stuber, G.D., Phillips, P.E., Wightman, R.M. \& Carelli, R.M. (2004) Dopamine operates as a subsecond modular of food seeking. J. Neurosci., 24, 1265-1271.

Salamone, J.D. (1991) Behavioral pharmacology of dopamine systems: a new synthesis. In Willner, P. \& Scheel-Kruder, J. (Eds), The Mesolimbic Dopamine System: from Motivation to Action. Wiley, Chichester, UK, pp. 598-613.

Salamone, J.D. (1992) Complex motor and sensorimotor functions of striatal and accumbens dopamine: involvement in instrumental behavior processes. Psychopharmacology, 107, 160-174.

Salamone, J.D., Aberman, J.E., Sokolowski, J.D. \& Cousins, M.S. (1999) Nucleus accumbens dopamine and rate of responding: neurochemical and behavioral studies. Psychobiology, 27, 236-247.

Salamone, J.D. \& Correa, M. (2002) Motivational views of reinforcement: implications for understanding the behavioral functions of nucleus accumbens dopamine. Behav. Brain Res., 137, 3-25.

Salamone, J.D., Correa, M., Mingote, S. \& Weber, S.M. (2003) Nucleus accumbens dopamine and the regulation of effort in food-seeking behavior: 
implications for studies of natural motivation, psychiatry, and drug abuse. J. Pharmacol. Exp. Ther, 305, 1-8.

Salamone, J.D., Cousins, M.S. \& Bucher, S. (1994) Anhedonia or anergia? Effects of haloperidol and nucleus accumbens dopamine depletion on instrumental response selection in a T-maze cost/benefit procedure. Behav. Brain Res., 65, 221-229.

Salamone, J.D., Cousins, M.D. \& Snyder, B.J. (1997) Behavioral functions of nucleus accumbens dopamine: empirical and conceptual problems with the anhedonia hypothesis. Neurosci. Biobehav. Rev., 21, 341-359.

Salamone, J.D., Kurth, P., McCullough, L.D. \& Sokolowski, J.D. (1995) The effects of nucleus accumbens dopamine depletions on continuously reinforced operant responding: contrasts with the effects of extinction. Pharmacol. Biochem. Behav., 50, 437-443.

Salamone, J.D., Kurth, P.A., McCullough, L.D., Sokolowski, J.D. \& Cousins, J.D. (1993a) The role of brain dopamine in response initiation: effects of haloperidol and regionally specific dopamine depletions on local rate of instrumental responding. Brain Res., 628, 218-226.

Salamone, J.D., Mahan, K. \& Rogers, S. (1993b) Ventrolateral striatal dopamine depletions impair feeding and food handling in rats. Pharmacol. Biochem. Behav., 44, 605-610.

Salamone, J.D., Steinpreis, R.E., McCullough, L.D., Grebel, D. \& Mahan, K. (1991) Haloperidol and nucleus accumbens dopamine depletion suppress lever pressing for food but increase free food consumption in a novel food choice procedure. Psychopharmacology, 104, 515-521.

Salamone, J.D., Wisniecki, A., Carlson, B.B. \& Correa, M. (2001) Nucleus accumbens dopamine depletions make animals highly sensitive to high fixed ratio requirements but do not impair primary food reinforcement. Neuroscience, 105, 863-870.

Sokolowski, J.D., Conlan, A. \& Salamone, J.D. (1998) A microdialysis study of nucleus accumbens core and shell dopamine during operant responding in the rat. Neuroscience, 86, 1001-1009.
Sokolowski, J.D. \& Salamone, J.D. (1998) The role of accumbens dopamine in lever pressing and response allocation: effects of 6-OHDA injected into core and dorsomedial shell. Pharmacol. Biochem. Behav., 59, 557-566.

Staddon, J.E.R. (1979) Operant behavior as adaptation to constraint. J. Exp. Psychol. Gen., 108, 48-67.

Taylor, J.R. \& Robbins, T.W. (1986) 6-Hydroxydopamine lesions of the nucleus accumbens, but not of the caudate nucleus, attenuate enhanced responding with reward-related stimuli produced by intra-accumbens D-amphetamine. Psychopharmacology, 90, 390-397.

Ungerstedt, U. (1971) Adipsia and aphagia after 6-hydroxydopamine induced degeneration of the nigro-striatal dopamine system. Acta Physiol. Scand. Suppl., 367, 95-122.

Wakabayashi, K.T., Fields, H.L. \& Nicola, S.M. (2004) Dissociation of the role of nucleus accumbens dopamine in responding to reward-predictive cues and waiting for reward. Behav. Brain Res., 154, 19-30.

Walton, M.E., Bannerman, D.M. \& Rushworth, M.F. (2002) The role of rat medial frontal cortex in effort-based decision making. J. Neurosci., 22, 10996-11003.

Wise, R. (2004) Dopamine, learning and motivation. Nat. Rev. Neurosci., 5, 483-494.

Wolterink, G., Van Zanten, E., Kamsteeg, H., Radhakishun, F.S. \& Van Ree, J.M. (1990) Functional recovery after destruction of dopamine systems in the nucleus accumbens of rats. I. Behavioral and biochemical studies. Brain Res., 507, 92-100.

Wyvell, C.L. \& Berridge, K.C. (2000) Intra-accumbens amphetamine increases the conditioned incentive salience of sucrose reward: enhancement of reward "wanting" without enhanced "liking" or response reinforcement. $J$. Neurosci., 20, 8122-8130.

Zigmond, M.J., Acheson, A.L., Stachowiak, M.K. \& Stricker, E.M. (1984) Neurochemical compensation after nigrostriatal bundle injury in an animal model of parkinsonism. Arch. Neurol., 41, 856-861. 